\title{
A homologous series of structures on the surface of $\mathrm{SrTiO}_{3}(110)$
}

\author{
James A. Enterkin ${ }^{1,2 \star}$, Arun K. Subramanian ${ }^{3 \dagger}$, Bruce C. Russell ${ }^{4}$, Martin R. Castell ${ }^{4}$, \\ Kenneth R. Poeppelmeier ${ }^{1,2}$ and Laurence D. Marks ${ }^{2,3}$
}

\begin{abstract}
Strontium titanate is seeing increasing interest in fields ranging from thin-film growth to water-splitting catalysis and electronic devices. Although the surface structure and chemistry are of vital importance to many of these applications, theories about the driving forces vary widely, ${ }^{1,2}$. We report here a solution to the $3 \times 1 \mathrm{SrTiO}_{3}(110)$ surface structure obtained through transmission electron diffraction and direct methods, and confirmed through density functional theory calculations and scanning tunnelling microscopy images and simulations, consisting of rings of six or eight cornersharing $\mathrm{TiO}_{4}$ tetrahedra. Further, by changing the number of tetrahedra per ring, a homologous series of $n \times 1(n \geq 2)$ surface reconstructions is formed. Calculations show that the lower members of the series $(n \leq 6)$ are thermodynamically stable and the structures agree with scanning tunnelling microscopy images. Although the surface energy of a crystal is usually thought to determine the structure and stoichiometry, we demonstrate that the opposite can occur. The $n \times 1$ reconstructions are sufficiently close in energy for the stoichiometry in the near-surface region to determine which reconstruction is formed. Our results indicate that the rules of inorganic coordination chemistry apply to oxide surfaces, with concepts such as homologous series and intergrowths as valid at the surface as they are in the bulk.
\end{abstract}

The structure of $\mathrm{SrTiO}_{3}$ is a cubic close-packed lattice of strontium and oxygen with strontium at the corners and oxygen at the face centres, and titanium at the body centres occupying those octahedral holes that are surrounded only by oxygen. Along the (110) direction $\mathrm{SrTiO}_{3}$ is polar, composed of alternating layers of $\mathrm{SrTiO}^{4+}$ and $\mathrm{O}_{2}{ }^{4-}$, that is, alternating layers with uncompensated nominal valence charges of $4+/ 4-$. In a fully ionic model, this leads to an unbalanced macroscopic dipole and infinite surface energy. Therefore, we expect a (110) surface to have a nominal excess surface valence of either $2+$ or $2-$ per surface unit cell, as otherwise energetically unfavourable holes in the valence band or electrons in the conduction band would be formed. There has been extensive discussion of the mechanisms of this 'charge compensation' for polar oxide surfaces in the literature (see for instance refs 3-5 and references therein). Various theories, such as a reduction of Coulomb forces ${ }^{2}$ or a minimization of 'dangling bonds' ${ }^{\prime}$, have been described as the driving force behind surface structure formation. An alternative model for oxide surfaces, first proposed for the $\mathrm{SrTiO}_{3}(001) 2 \times 1$ surface ${ }^{6}$, is that the rules of inorganic coordination chemistry dominate, although, as the (001) surface is not polar, we might question the generality of this model.

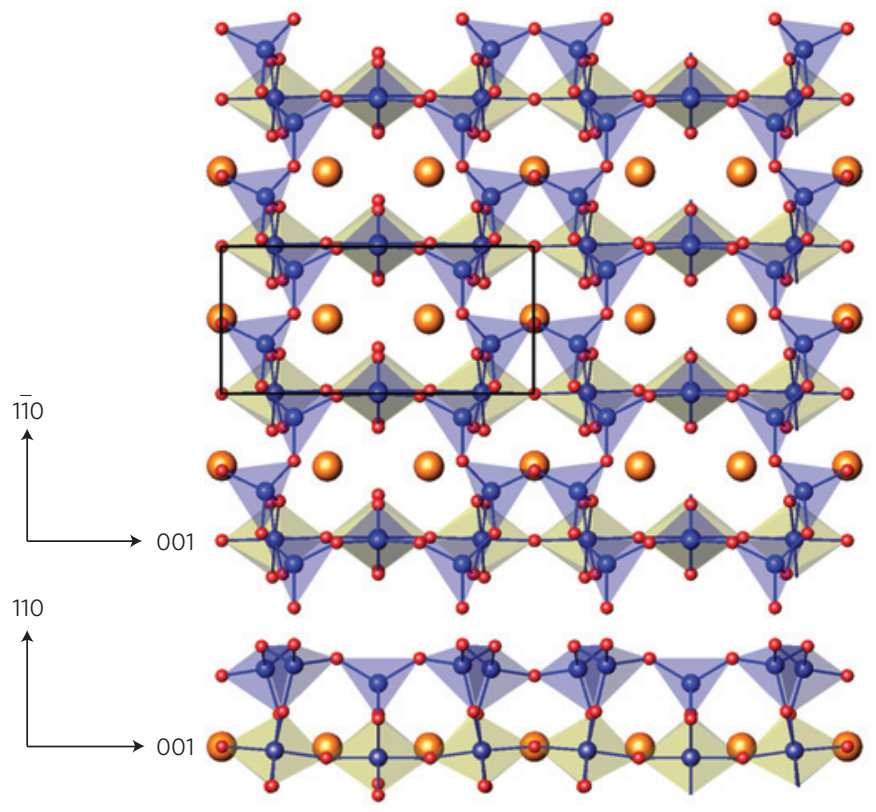

Figure 1 | The surface structure of the $3 \times 1$ reconstruction and the top bulk layer. Surface $\mathrm{TiO}_{4}$ tetrahedra are shown in blue, bulk $\mathrm{TiO}_{6}$ octahedra in yellow, oxygen anions in red and strontium cations in orange. Top: a view perpendicular to the surface with the unit cell outlined in black. Bottom: a view parallel to the surface.

For the $\mathrm{SrTiO}_{3}(110)$ surface under oxidizing conditions, several surface reconstructions have been observed, including $3 \times 1$, $4 \times 1,5 \times 1$ and $6 \times 1$ reconstructions ${ }^{7}$, which appear to be related. A reconstruction on the (110) surface is termed $n \times m$, indicating that it has dimensions of $n$ times the bulk unit-cell length in the [001] direction and $m$ times the bulk unit-cell length in the [110] direction. These $n \times 1(n=3-6)$ reconstructions show similar features in scanning tunnelling microscopy (STM), and can evolve from one to the next, often with two being present at the same time. Other reconstructions, including $2 \times 5$, $3 \times 4,4 \times 4,4 \times 7,6 \times 4$ (ref. 8) and $c(2 \times 6)$ (ref. 9), have been found following ultrahigh-vacuum annealing and seem to be unrelated. A number of theoretical studies have been carried out (see refs 10-13 and references therein), but with structures derived from bulk terminations that do not match the observed unit cells.

${ }^{1}$ Department of Chemistry, Northwestern University, Evanston, Illinois 60208, USA, ${ }^{2}$ Institute for Catalysis in Energy Processes, Northwestern University, Evanston, Illinois 60208, USA, ${ }^{3}$ Department of Materials Science and Engineering, Northwestern University, Evanston, Illinois 60208, USA, ${ }^{4}$ Department of Materials, University of Oxford, Parks Road, Oxford, OX13PH, UK. 'Present address: 2140 Research Drive, Livermore, California 94550, USA.

*e-mail: J-Enterkin@northwestern.edu. 


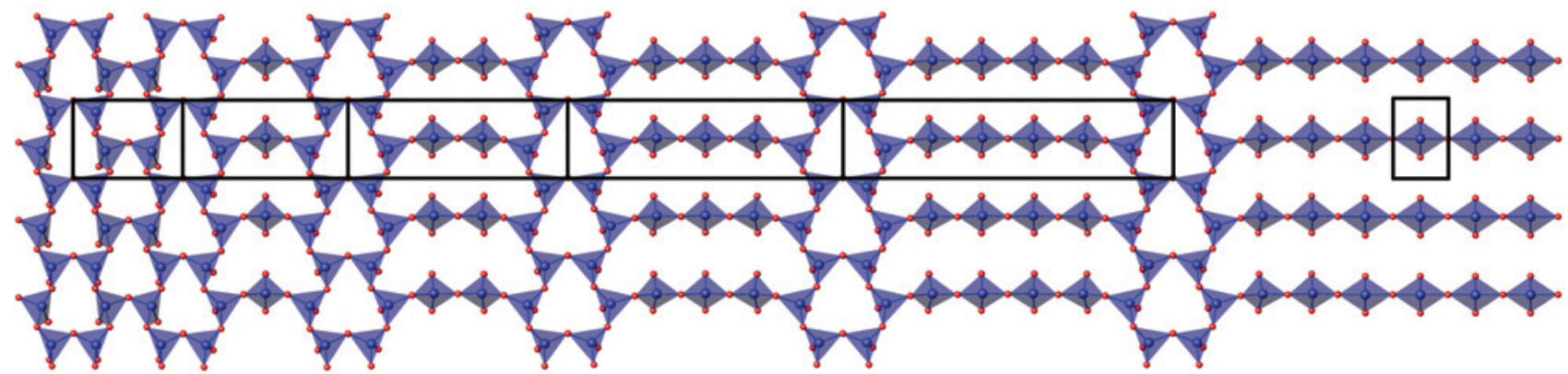

Figure 2 | The surface structures of calculated members of the homologous series of $\boldsymbol{n} \times \mathbf{1}$ surface structures viewed perpendicular to the surface. For clarity, the bulk is omitted. $\mathrm{TiO}_{4}$ tetrahedra are shown in blue, with oxide anions in red. Unit cells are outlined in black; from left to right $n=2,3,4,5,6, \infty$. As $n$ increases, an extra $\mathrm{TiO}_{4}$ tetrahedron is inserted into the sides of the larger ring. The newly inserted tetrahedron has coordination similar to that of the tetrahedron at the middle of the eight-member ring in the $3 \times 1$ structure: it corner shares with two other surface $\mathrm{TiO}_{4}$ tetrahedra, and edge shares with a single bulk $\mathrm{TiO}_{6}$ octahedron. For $n=\infty$ all $\mathrm{TiO}_{4}$ tetrahedra are of this type.

Annealing of single-crystal (110)-oriented transmission electron microscopy samples at $950^{\circ} \mathrm{C}$ yielded a diffraction pattern with streaking in the [110] direction. Increasing the temperature to $1,000{ }^{\circ} \mathrm{C}$ yielded a three-fold ordering along the [001] direction, while the streaking along the [110] direction remained. Further increasing the temperature to $1,100^{\circ} \mathrm{C}$ enabled the surface to fully order, with a combination of $3 \times 1$ and $1 \times 4$ (see Supplementary Fig. S1) reconstructions, as reported previously ${ }^{7}$. The $3 \times 1$ was the main phase, with the $1 \times 4$ present only near the step edges (as also observed by STM; ref. 7), and only the $3 \times 1$ is analysed here.

Transmission electron diffraction is a powerful tool for solving surface structures and refining atomic positions. As only amplitudes are recorded and the phase information is lost, we use direct methods (see refs 14, 15 and references therein) to obtain plausible solutions. The structure was solved and refined in $p 1 \mathrm{~m} 1$ plane group symmetry showing rings of six or eight strong scattering features (see Supplementary Fig. S2). These were found to be titanium atom sites, and using a combination of difference maps and least-squares refinement the oxygen sites were found. To obtain further information, including atomic positions normal to the surface, density functional theory (DFT) calculations were carried out using the Wien $2 \mathrm{k}$ code $\mathrm{e}^{16}$. These confirmed that the structure was stable and of low energy, as will be discussed further below. We also simulated the STM images for this structure, which were comparable to experimental results (see later). Other structural possibilities were examined: all had significantly inferior refinement, did not match the STM images and were much higher in energy.

The surface structure (Fig. 1) is composed of corner-sharing $\mathrm{TiO}_{4}$ tetrahedra, arranged into six- and eight-member rings. The tetrahedra in the six-member rings all corner share with three other surface tetrahedra and one subsurface $\mathrm{TiO}_{6}$ octahedron. The titanium tetrahedron in the middle of the eight-member ring, the only one that is not also part of a six-member ring, is corner sharing with two other surface tetrahedra, and edge sharing with one subsurface $\mathrm{TiO}_{6}$ octahedron. The composition of the surface is $\mathrm{Ti}_{5 / 3} \mathrm{O}_{13 / 3}{ }^{2-}$ per $1 \times 1$ unit cell, valence compensating the surface.

As already mentioned, the $3 \times 1$ reconstruction is only one member of a series of $n \times 1$ reconstructions. We can readily extend from the $3 \times 1$ to a homologous series by varying the size of the larger ring as illustrated in Fig. 2: reducing it to $\mathrm{six} \mathrm{TiO}_{4}$ tetrahedra gives a $2 \times 1$ reconstruction; increasing to ten, a $4 \times 1$ reconstruction is formed. All these reconstructions are fully valence compensated with nominal surface compositions of $\mathrm{Ti}_{(n+2) / n} \mathrm{O}_{(3 n+4) / n}{ }^{2-}$ per $1 \times 1$ unit cell for all of the $n \times 1$ reconstructions, differing in the surface excess of $\mathrm{TiO}_{2}$. These structures were optimized through DFT for $2 \leq n \leq 6$ and $n=\infty$, and the energetics are summarized in Fig. 3. A convex-hull construction implies that (within theoretical

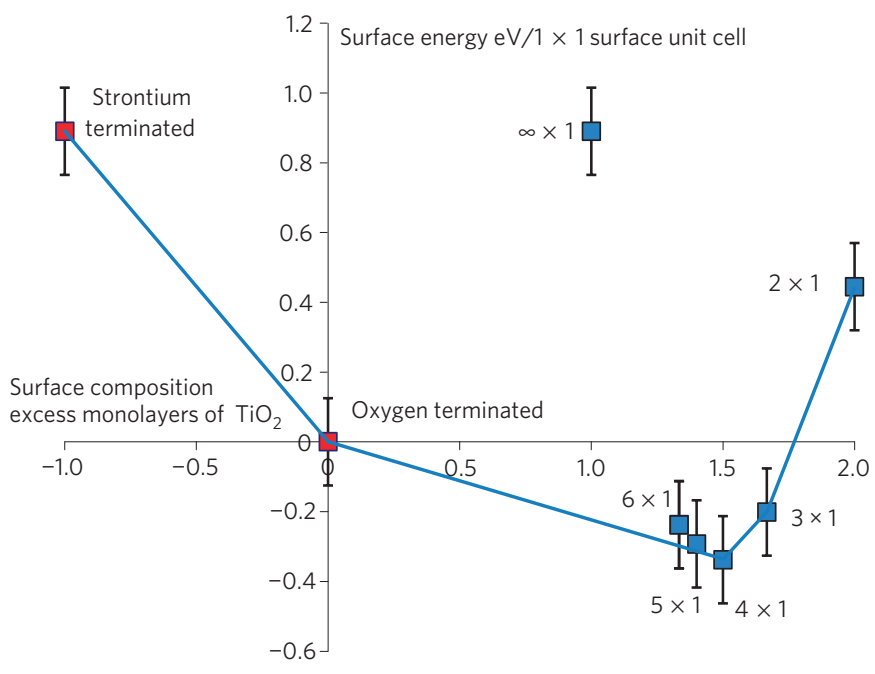

Figure 3 | A plot of the surface energies for $n \times 1$ surfaces and several previously proposed surfaces. The convex hull construction (blue line) shows the stable surface for a given composition. The vertical axis is the surface energy in electronvolts per $1 \times 1$ surface unit cell. The horizontal axis is the composition in terms of excess monolayers of $\mathrm{TiO}_{2}$ at the surface; negative numbers indicate an excess of $\mathrm{SrO}$. Error bars were estimated on the basis of the differences in energies using various functionals ${ }^{20}$.

error) the structures lying on the hull should appear for different surface compositions, agreeing with the experimental observations. As an independent check we simulated STM images and compared them with experimental images of $3 \times 1,4 \times 1,5 \times 1$ and $6 \times 1$ reconstructions (see Fig. 4 and Supplementary Fig. S3). These images and simulations correlate with one another in all major aspects. Note that the STM images indicate that layers of (for instance) $4 \times 1$ can occur as intergrowths in a matrix of $3 \times 1$, a point we shall return to later.

To form a specific structure, the structure must be both thermodynamically stable and kinetically accessible. Although kinetics is beyond the scope of the present work, we can draw some conclusions with respect to the thermodynamic stability. The most important factors contributing to the stability of the surfaces in this homologous series seems to be maintaining acceptable coordination environments combined with valence compensation and increased covalence at the surface.

For the whole series, the coordination of the outermost titanium is tetrahedral. Just as $\mathrm{SiO}_{2}$ can form an octahedral phase isostructural with rutile under pressure, it is straightforward to show through DFT calculations that tetrahedrally coordinated $\mathrm{TiO}_{2}$ 


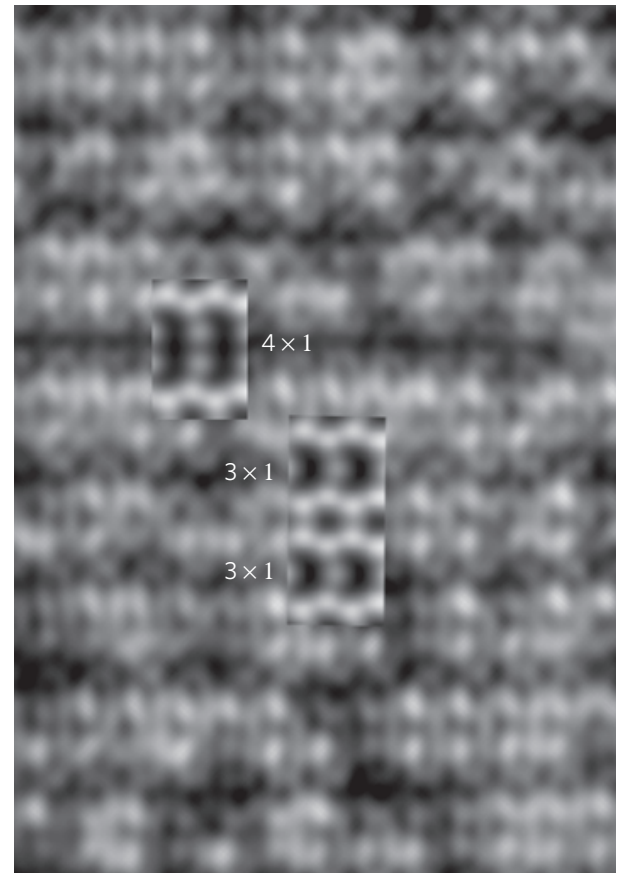

Figure 4 | An STM image of a $3 \times 1$ surface with an intergrowth of $4 \times 1$ with simulations overlaid. Sample bias $=0.9 \mathrm{~V}$, tunnelling current $=0.4 \mathrm{nA}$.

is not significantly higher in energy than rutile and would be more stable under negative pressure (see Supplementary Fig. S4); in fact, tetrahedral coordination has been observed on the (001) surface of $\mathrm{TiO}_{2}$ (ref. 17). The bond-valence sums (see Supplementary Table S1), a commonly used coordination metric in the bulk, show that the more stable structures have coordination similar to that of the bulk. The bond-valence sum for the titanium is very close to that of the bulk (within 5\%) for $n=3,4$, slightly less for $n=2,5,6, \infty$ (all 8-18\% less than bulk). For the surface oxygen, the bond-valence sum is nearly identical to that of the bulk for $n=2,3$, and decreases as $n$ increases, remaining close to that of the bulk (within 15\%) until $n=6$, but dropping drastically for $n=\infty$ (38\% less than that of the bulk). By this metric the coordination for $n=2-6$ is comparable to that of bulk $\mathrm{SrTiO}_{3}$, whereas that for $n=\infty$ is not; the DFT energetics indicate that $n=\infty$ is not a preferred structure.

With the reduced coordination, the non-bonded repulsions between the oxygen atoms are decreased, enabling shorter and more covalent bonds. The decreased ionicity can be readily seen through the bond distances and Bader charges, in addition to bond-valence sums (see Supplementary Table S1). The bonds at the surface are, on the whole, slightly shorter and more covalent than in the bulk structure: for $n=3$, the average $\mathrm{Ti}-\mathrm{O}$ bond length for the surface structure is $1.814 \AA$, as compared with the $1.953 \AA$ bulk Ti-O bond distance. The Bader charges indicate that the surface species are less charged than the bulk, with the average Bader charge for an oxygen atom in the $n=3$ structure increasing from -1.18 at the surface through -1.26 at the linking $\mathrm{O}_{2}$ layer (oxygen bonded to both surface and subsurface titanium) and -1.29 in the first subsurface SrTiO layer to $\sim-1.31$ in deeper layers. Similarly, the Bader charge for a titanium atom increases from 2.33 at the surface to 2.36 at the first subsurface SrTiO layer and $\sim 2.37$ in deeper layers.

The trends across the series are straightforward: the charge is lower and the bonds are shorter at the surface, and both increase with increasing distance from the surface. The degree of this decreased charge at the surface changes only slightly from $n=2$ to $n=6$, but in the $n=\infty$ structure the charge on the surface titanium is significantly less, whereas that on the first subsurface titanium is significantly higher than those deeper in the structure. The charge on the surface oxygen is also decreased by as much as 0.2 electrons for $n=2$, and as $n$ increases this difference decreases to 0.03 electrons by $n=\infty$. For $n=3$ to $n=6$ the charge on oxygen atoms then increases gradually deeper in the structure. For $n=2$ the charge on the linking $\mathrm{O}_{2}$ layer is greater than the charge on the oxygen in the first subsurface SrTiO layer, whereas for $n=\infty$ the charge on the surface oxygen exceeds that in either the linking $\mathrm{O}_{2}$ layer or the first subsurface SrTiO layer. This indicates that the $n=3$ to $n=6$ structures, and to a lesser extent the $n=2$ structure, successfully minimize the charge at the surface, whereas the unstable $n=\infty$ structure does not.

Can we start to predict oxide surface structures? We believe the answer is that we can. Obviously the thermodynamic structures will in almost all cases (particularly under oxidizing conditions) be valence compensated, but this is not enough; they will have polyhedral coordination chemistry similar to that of the bulk, with metrics such as bond-valence sums equally useful. The way to understand oxide surfaces is not to look out towards the vacuum, but to look in towards the bulk. Homologous series are well known in bulk oxides, and if the STM images were high-resolution electron microscopy images of a bulk oxide the fact that they show intergrowths would be relatively unremarkable; the fact that this is occurring in a single monolayer is however remarkable, being the first example of this important concept for bulk oxides occurring at a surface. We believe that a century of inorganic chemistry can guide us to predict oxide surface structures.

\section{Methods}

Diffraction analysis. Starting with a (110)-oriented $\mathrm{SrTiO}_{3}$ single-crystal wafer, samples were prepared by standard solid-state transmission electron microscopy sample preparation techniques: $3 \mathrm{~mm}$ disks were cut with a rotary cutter, mechanically polished to a thickness of about $120 \mu \mathrm{m}$ and dimpled, then ion milled with $\mathrm{Ar}^{+}$ions in a Gatan precision ion-polishing system to produce electrontransparent samples. Samples were annealed in a tube furnace with flowing highpurity oxygen. Electron-diffraction patterns were obtained using a Hitachi UHV9000 electron microscope at Northwestern University. A series of exposures was taken covering the entire dynamic range ${ }^{18}$, then digitized to eight bits with a $25 \mu \mathrm{m}$ pixel size using an Optronics P-1000 microdensitometer, and intensities extracted using a cross-correlation technique ${ }^{19}$. The intensities were averaged using a $p 2 \mathrm{~mm}$ Patterson plane group symmetry, yielding 36 independent reflections. Under the exposure conditions used, the intensity readout from the microdensitometer was proportional to the true intensities of the diffraction spots. Beam damage was checked by comparing early exposures with exposures under identical conditions taken later on, and, as expected for strontium titanate, found to be negligible. As a glide plane was incommensurate with the bulk termination, only $p 11 \mathrm{~m}, \mathrm{p} 1 \mathrm{~m} 1$ or $p 2 \mathrm{~mm}$ plane group symmetries were possible. Direct methods (see refs 14, 15 and references therein) were used for all possible plane groups to obtain plausible solutions. Final structure refinement was carried out on the basis of both $R^{2}$ and $\chi^{2}$, with the best results found in plane group $p 1 m 1$ with $R=0.07066$ and $\chi^{2}=2.64$.

DFT calculations. DFT calculations were carried out using the Wien $2 \mathrm{k}$ code ${ }^{16}$, with, for improved accuracy, PBE0 and TPSSh on-site functionals as described elsewhere, with errors estimated from the differences between the functionals ${ }^{20}$. An RKmax parameter of 6.12 was used with RMTs of 2.36, 1.72 and 1.54 for strontium, titanium and oxygen, respectively. Calculations were carried out using five bulk $\mathrm{SrTiO}^{4+}$ layers separated by four bulk $\mathrm{O}_{2}{ }^{4-}$ layers, with a $\mathrm{Ti}_{(n+2) / n} \mathrm{O}_{(3 n+4) / n}{ }^{2-}$ surface on either side. This was found to be thick enough, as the innermost $\mathrm{SrTiO}^{4+}$ layer was only minimally distorted. STM images were simulated using a modified Tersoff-Hamann approximation ${ }^{21}$, considering electrons from the Fermi energy $\left(E_{\mathrm{f}}\right)$ to $1.0 \mathrm{eV}$ above the conduction band edge.

Received 24 August 2009; accepted 20 January 2010; published online 14 February 2010

\section{References}

1. Pashley, M. D. Electron counting model and its application to island structures on molecular-beam epitaxy grown $\mathrm{GaAs}(001)$ and $\mathrm{ZnSe}(001)$. Phys. Rev. B 40, 10481-10487 (1989).

2. Wolf, D. Reconstruction of $\mathrm{NaCl}$ surfaces from a dipolar solution to the Madelung problem. Phys. Rev. Lett. 68, 3315-3318 (1992).

3. Goniakowski, J., Finocchi, F. \& Noguera, C. Polarity of oxide surfaces and nanostructures. Rep. Prog. Phys. 71, 016501 (2008). 
4. Deak, D. S. Strontium titanate surfaces. Mater. Sci. Technol. 23, 127-136 (2007)

5. Noguera, C. Polar oxide surfaces. J. Phys. Condens. Matter 12, R367-R410 (2000).

6. Erdman, N. et al. The structure and chemistry of the $\mathrm{TiO}_{2}$-rich surface of $\mathrm{SrTiO}_{3}(001)$. Nature 419, 55-58 (2002).

7. Russell, B. C. \& Castell, M. R. Reconstructions on the polar $\mathrm{SrTiO}_{3}(110)$ surface: Analysis using STM, LEED, and AES. Phys. Rev. B 77, 245414 (2008).

8. Brunen, J. \& Zegenhagen, J. Investigation of the $\mathrm{SrTiO}_{3}(110)$ surface by means of LEED, scanning tunnelling microscopy and Auger spectroscopy. Surf. Sci. 389, 349-365 (1997)

9. Bando, H. et al. Structure and electronic states on reduced $\mathrm{SrTiO}_{3}(110)$ surface observed by scanning-tunnelling-microscopy and spectroscopy. J. Vac. Sci. Technol. B 13, 1150-1154 (1995).

10. Bottin, F., Finocchi, F. \& Noguera, C. Facetting and $(n \times 1)$ reconstructions of $\mathrm{SrTiO}_{3}(110)$ surfaces. Surf. Sci. 574, 65-76 (2005).

11. Heifets, E. et al. Ab initio calculations of the $\mathrm{SrTiO}_{3}(110)$ polar surface. Phys. Rev. B 69, 035408 (2004).

12. Kotomin, E. A. et al. Comparative study of polar perovskite surfaces. Surf. Sci. 566, 231-235 (2004)

13. Eglitis, R. I. \& Vanderbilt, D. First-principles calculations of atomic and electronic structure of $\mathrm{SrTiO}_{3}(001)$ and (011) surfaces. Phys. Rev. B 77, 195408 (2008).

14. Marks, L. D., Sinkler, W. \& Landree, E. A feasible set approach to the crystallographic phase problem. Acta Crystallogr. Sect. A 55, 601-612 (1999).

15. Chiaramonti, A. N. \& Marks, L. D. Atomic resolution transmission electron microscopy of surfaces. J. Mater. Res. 20, 1619-1627 (2005).

16. Blaha, P. et al. WIEN2k, An Augmented Plane Wave Plus Local Orbitals Program for Calculating Crystal Properties (Technical Univ. Vienna, 2001).

17. Warschkow, O. et al. Structure and local-equilibrium thermodynamics of the $c(2 \times 2)$ reconstruction of rutile $\mathrm{TiO}_{2}(100)$. Phys. Rev. Lett. 100, 86102 (2008).
18. Jayaram, G., Xu, P. \& Marks, L. D. Structure of Si(100)-(2×1) surface using UHV transmission electron-diffraction. Phys. Rev. Lett. 71, 3489-3492 (1993).

19. Xu, P., Jayaram, G. \& Marks, L. D. Cross-correlation method for intensity measurement of transmission electron-diffraction patterns. Ultramicroscopy 53, 15-18 (1994)

20. Marks, L. D., Chiaramonti, A., Tran, F. \& Blaha, P. The small unit cell reconstructions of $\mathrm{SrTiO}_{3}$ (111). Surf. Sci. 603, 2179-2187 (2009).

21. Tersoff, J. \& Hamann, D. R. Theory of the scanning tunnelling microscope. Phys. Rev. B 31, 805-813 (1985).

\section{Acknowledgements}

This work was supported by the Northwestern University Institute for Catalysis in Energy Processing, funded through the US Department of Energy, Office of Basic Energy Science (award number DE-FG02-03-ER15457, J.A.E., K.R.P. and L.D.M.). We also acknowledge funding on NSF DMR-0455371/001 (A.K.S.), DOE DE-FG02-01ER45945 (L.D.M. and computer hardware) and NSF DMR-0710643 (B.C.R., M.R.C., L.D.M. and computer hardware)

\section{Author contributions}

A.K.S. obtained the transmission electron diffraction patterns. B.C.R. and M.R.C obtained the STM images. J.A.E. and L.D.M. carried out the DFT calculations. J.A.E., with contributions from L.D.M. and K.R.P., carried out direct methods, structure solution and analysis of the homologous series. J.A.E. wrote the manuscript, which M.R.C., K.R.P. and L.D.M. edited

\section{Additional information}

The authors declare no competing financial interests. Supplementary information accompanies this paper on www.nature.com/naturematerials. Reprints and permissions information is available online at http://npg.nature.com/reprintsandpermissions. Correspondence and requests for materials should be addressed to J.A.E. 